\title{
SOME ELEMENTARY PROOFS OF BASIC THEOREMS IN THE COHOMOLOGY OF QUASI-COHERENT SHEAVES
}

\author{
GEORGE R. KEMPF
}

In his fundamental paper [5], Serre initiated the study of the Čech cohomology of coherent sheaves on (separated) algebraic varieties with their Zariski topology. He proved that coherent sheaves on affine varieties have zero higher cohomology groups. Using this theorem, he showed that one may compute the cohomology of coherent sheaves by using the more down-to-earth Čech cohomology of any affine open covering of the algebraic variety.

Grothendieck [2] defined a cohomology theory of sheaves on any topological space by injective resolutions. In this theory, the emphasis is on the long exact sequence of cohomology arising from any short exact sequence of sheaves. A modification of this theory was presented by Godement [1] making use of canonical flabby resolutions.

In algebraic geometry, Grothendieck [3] greatly extended Serre's results to quasi-coherent sheaves on schemes. His argument appears to be a direct translation of Serre's to the more general context and freely employs spectral sequences.

In the first section of this paper, I will give a proof of Serre's affine vanishing theorem. My proof of this theorem makes no use of the Čech cohomology and deals with the general cohomology theory. The main step is a direct proof of the effaceability of the cohomology of a quasicoherent sheaf on an affine scheme. I prefer to reserve Čech cohomology for its proper place as one of many possible ways of computing the sheaf cohomology.

In section two, I develop formalities about direct limits of sheaves on quasi-noetherian topological spaces. These are topological spaces with strong finiteness properties. Such spaces arise in algebraic geometry as the underlying spaces of quasi-compact, quasi-separated schemes; e.g. affine schemes. These simple formalities are used quite effectively to deduce the results of section three.

In section four, I show that the sheaf cohomology coincides with Čech cohomology in a reasonable situation. The same type of argument yields easily a special case of the Künneth formula.

After many propositions, I will refer to the closest approximation that 
I can find in E.G.A. III [3]. One implicit objective of this paper is the elimination of spectral sequence arguments in the proofs of these basic results. Lastly, I want to thank Columbia University for partly supporting the research in this paper.

A word of warning is that the "preschemes" of E.G.A. are now called "schemes". Furthermore, the "schemes" of E.G.A. are now called "separated schemes".

1. Serre's Vanishing Theorem. Let $\mathscr{F}$ be a sheaf on a topological space $X$. Let $U$ be an open subset of $X$. Denote by ${ }_{U} \mathscr{F}$ the sheaf gotten by setting $\Gamma\left(V,{ }_{U} \mathscr{F}\right)=\Gamma(V \cap U, \mathscr{F})$ for any open subset $V$ of $X$. Thus, ${ }_{U} \mathscr{F}$ is the image under the inclusion $U \subset X$ of the restriction $\left.\mathscr{F}\right|_{U}$ of $\mathscr{F}$ to $U$. We have a natural mapping $\mathscr{F} \rightarrow{ }_{U} \mathscr{F}$.

For the remainder of this paper, I will assume that all sheaves are sheaves of abelian groups. A basis of a topological space will be a collection of arbitrarily small open subsets which is closed under finite intersections.

We now will develop a preliminary

Proposition 1. Let $\mathscr{F}$ be a sheaf on a space X. Assume that $X$ possesses a basis $\mathscr{U}$ such that $H^{i}\left(U,\left.\mathscr{F}\right|_{U}\right)$ is zero for $0<i<$ some positive integer $n$ for all $U$ in $\mathscr{U}$.

Given any element $\alpha$ of $H^{n}(X ; \mathscr{F})$, there is a covering $\mathscr{V}$ of $X$ by members of $\mathscr{U}$ such that the image of $\alpha$ in $H^{n}\left(X,{ }_{V} \mathscr{F}\right)$ is zero for all $V$ in $\mathscr{V}$.

Proof. Let $0 \rightarrow \mathscr{F} \rightarrow \mathscr{G} \rightarrow \mathscr{H} \rightarrow 0$ be a short exact sequence of sheaves where $\mathscr{G}$ is a flabby sheaf. Thus, $H^{i}\left(U,\left.\mathscr{G}\right|_{U}\right)=0$ for positive $i$ for any open subset $U$ of $X$. By the long exact sequence of cohomology, we have an exact sequence

( b ) $0 \rightarrow \Gamma\left(U,\left.\mathscr{F}\right|_{U}\right) \rightarrow \Gamma\left(U,\left.\mathscr{G}\right|_{U}\right) \rightarrow \Gamma\left(U,\left.\mathscr{H}\right|_{U}\right) \rightarrow H^{1}\left(U,\left.\mathscr{F}\right|_{U}\right) \rightarrow 0$,

and isomorphisms

( $\left.\begin{array}{l}\text { b } \\ \text { b }\end{array}\right) \quad H^{i}\left(U,\left.\mathscr{H}\right|_{U}\right) \underset{\sim}{\approx} H^{i+1}\left(U,\left.\mathscr{F}\right|_{U}\right)$ for $i>0$.

For any open subset $V$ of $X$, we have an exact commutative diagram

$$
\begin{aligned}
& 0 \rightarrow \mathscr{F} \rightarrow \mathscr{G} \rightarrow \mathscr{H} \rightarrow 0 \\
& 0 \rightarrow{ }_{V} \mathscr{F} \rightarrow{ }_{V} \mathscr{G} \rightarrow{ }_{V} \mathscr{H},
\end{aligned}
$$

where ${ }_{V} \mathscr{G}$ is a flabby sheaf. The image of $\mathscr{H}$ and the image ${ }_{V} \mathscr{G}$ in ${ }_{V} \mathscr{H}$ coincide: denote this image by $\mathscr{K}$. Repeating the above idea for the sequence, $0 \rightarrow{ }_{V} \mathscr{F} \rightarrow{ }_{v} \mathscr{G} \rightarrow \mathscr{K} \rightarrow 0$, we have an exact sequence

$$
0 \rightarrow \Gamma\left(X,{ }_{V} \mathscr{F}\right) \rightarrow \Gamma\left(X,{ }_{V} \mathscr{G}\right) \rightarrow \Gamma(X, \mathscr{K}) \rightarrow H^{1}\left(X,{ }_{V} \mathscr{F}\right) \rightarrow 0,
$$

and isomorphisms 


$$
H^{i}(X, \mathscr{K}) \stackrel{\approx}{\rightarrow} H^{i+1}\left(X, V^{\mathscr{F}}\right) \text { for } i>0 .
$$

The ( $b$ ) lines for $U=X$ are parallel to the (\#) lines. Also, we have an inclusion, $\Gamma(X, \mathscr{K}) \subseteq \Gamma(X, v \mathscr{H})$.

Assume first that $n>1$. Then, if $V$ and $W$ are members of $\mathscr{U}$, the sequence (b) for $U=V \cap W$ shows that $\Gamma\left(U, v^{\mathscr{G}}\right) \rightarrow \Gamma(U, v \mathscr{H})$ is surjective. Hence, $\mathscr{K}={ }_{v} \mathscr{H}$. Furthermore, $\left(\begin{array}{ll}b & b\end{array}\right)$ shows that $\mathscr{H}$ satisfies the assumption as in the proposition for the integer $n-1$. The parallel isomorphisms $(\# \#)$ may then be used for the inductive step.

It remains to consider the case when we have assumed nothing; i.e. $n=1$. By the ( $b$ ) sequence for $U=X$, our element $\alpha$ of $H^{1}(X, \mathscr{F})$ is $\delta(\beta)$ for some element $\beta$ of $\Gamma(X, \mathscr{H})$. By (\#), the image of $\alpha$ in $H^{1}\left(X,{ }_{v} \mathscr{F}\right)$ is zero $\Leftrightarrow$ the image of $\beta$ in $\Gamma(X, \mathscr{K})$ (or $\Gamma\left(X,{ }_{V} \mathscr{H}\right)=\Gamma(V, \mathscr{H})$ ) lifts to an element of $\Gamma\left(X, V_{G}\right)=\Gamma(V, \mathscr{G})$. As $\mathscr{U}$ consists of arbitrarily small open subsets, there is no problem finding a subcovering $\mathscr{V}$ satisfying the last condition because $\mathscr{G} \rightarrow \mathscr{H} \rightarrow 0$ is exact.

We are ready to prove the first key theorem on the cohomology of schemes. This is Serre's

THEOREM 2. $[3,1.3 .1]$. Let $\mathscr{F}$ be a quasi-coherent sheaf on an affine scheme $X$. Then, $H^{i}(X, \mathscr{\mathscr { P }})$ is zero if $i>0$.

Proof. Let $\mathscr{U}$ be the basis of $X$, consisting of affine open subsets $X_{f}$ for $f \in \Gamma\left(X, \mathscr{O}_{X}\right){ }_{X_{f}} \mathscr{F}$ is the quasi-coherent sheaf $\Gamma(X, \mathscr{F})_{f}$. By induction, we may assume $H^{i}(X, \mathscr{F})$ is zero for $0<i<n$ for all quasi-coherent sheaves $\mathscr{F}$ on affine schemes $X$. The hypothesis of Proposition 1 is verified for the covering $\mathscr{U}$. Therefore, for any given element $\alpha$ of $H^{n}(X, \mathscr{F})$, we may find a covering $V_{1}, \ldots, V_{p}$ of $X$ by members of $\mathscr{U}$ such that the image of $\alpha$ in

$$
H^{n}\left(X, \underset{1 \leq j \leq p}{\oplus} V_{i}^{\mathscr{F}}\right) \text { is zero. }
$$

Hence the long exact sequence of cohomology of the short exact sequence of quasicoherent sheaves

$$
0 \rightarrow \mathscr{F} \rightarrow \oplus_{v_{j}} \mathscr{F} \rightarrow \mathscr{G} \rightarrow 0,
$$

shows that $\alpha$ is in the image $\delta\left(H^{n-1}(X, \mathscr{G})\right.$. If $n>1, H^{n-1}(X, \mathscr{G})$ is zero by the inductive assumption. If $n=1, \delta$ is zero because $\Gamma(X,-)$ is exact for quasi-coherent sheaves. In any case, $\alpha$ is zero.

Thus, $H^{n}(X, \mathscr{F})$ is zero for all positive $n$.

COROLlaRY 3. [3, 1.3.3] Let $f: X \rightarrow Y$ be an affine morphism of schemes and $\mathscr{F}$ a quasi-coherent sheaf on $X$. Then, there is a natural isomorphism

$$
H^{i}(X, \mathscr{F}) \cong H^{i}\left(Y, f_{*} \mathscr{F}\right)
$$

Proof. Let $\mathscr{F} \rightarrow \mathscr{F}^{*}$ be a flabby resolution of $\mathscr{F}$. Let $V$ be an open 
affine subscheme of $X$. As $f$ is an affine morphism, then $f^{-1} V$ is an open affine subscheme of $X$. By Theorem 2 applied to the quasi-coherent sheaf $\mathscr{F}$ restricted to $f^{-1} V$, the complex

$$
0 \rightarrow \Gamma\left(f^{-1} V, \mathscr{F}\right) \rightarrow \Gamma\left(f^{-1} V, \mathscr{F}^{*}\right)
$$

is exact. As the $V$ 's are arbitrarily small, this shows that $f_{*} \mathscr{F} \rightarrow f_{*} \mathscr{F}^{*}$ is a flabby resolution of $f_{*} \mathscr{F}$.

As $\Gamma\left(Y, f_{*} \mathscr{G}\right)$ is naturally isomorphic to $\Gamma(X, \mathscr{G})$ for any sheaf $\mathscr{G}$ on $X$, the two complexes, $\Gamma\left(Y, f_{*} \mathscr{F}^{*}\right)$ and $\Gamma\left(X, \mathscr{F}^{*}\right)$, are naturally isomorphic and, hence, have isomorphic cohomology groups. On the other hand, their $i^{\text {th }}$ cohomology groups are actually isomorphic to

$$
H^{i}\left(Y, f_{*} \mathscr{F}\right) \text { and } H^{i}(X, \mathscr{F}) \text { respectfully. }
$$

2. Direct Limits of Sheaves. The purpose of this section is to develop some simple properties of sheaves on a special type of topological space, which arises in algebraic geometry. My development is parallel to Godement's treatise [1]. Unfortunately, these spaces are only given marginal treatment by him. I will give a detailed discussion as I want to prove Theorem 8 in a slightly more general form than he gives.

The topological spaces of interest are called quasi-noetherian. A space is quasi-noetherian if it possesses a basis (closed under finite intersection) of quasi-compact open subsets and is itself quasi-compact. In E.G.A., a space is noetherian if all of its open subsets are quasi-compact. In Godement's terminology, these later spaces are called spaces of Zariski.

It will be necessary to generalize the notion of flabby sheaf. A sheaf on a space $X$ is quasi-flabby if the restriction $\Gamma(X, \mathscr{F}) \rightarrow \Gamma(U, \mathscr{F})$ is always surjective for any quasi-compact open subset $U$ of $X$. On a quasinoetherian space, the quasi-flabby sheaves possess most of the pleasant properties enjoyed by flabby sheaves.

We begin our step-by-step checking of these pleasant properties by modifying Godement's theorem 3.1.2. The modified proof does not even need Zorn's lemma. The result in question is

Proposition 4. Let $0 \rightarrow \mathscr{L}^{\prime} \rightarrow \mathscr{L} \rightarrow \mathscr{L}^{\prime \prime} \rightarrow 0$ be an exact sequence of sheaves on a quasi-noetherian space $X$. If $\mathscr{L}^{\prime}$ is quasi-flabby, then the sequence

$$
0 \rightarrow \Gamma\left(X, \mathscr{L}^{\prime}\right) \rightarrow \Gamma(X, \mathscr{L}) \rightarrow \Gamma\left(X, \mathscr{L}^{\prime \prime}\right) \rightarrow 0
$$

is exact.

Proof. Let $s$ be a section of $\mathscr{L}^{\prime \prime}$. Consider the family $\mathscr{V}$ of quasicompact open subsets $V$ such that $\left.s\right|_{V}$ is represented by an element of $\Gamma(V, \mathscr{L})$. We need to see that $X$ is contained in $\mathscr{V}$. As $\mathscr{V}$ covers $X$ and $X$ is 
quasi-compact, it will suffice to show that $\mathscr{V}$ is closed under double (hence, finite) unions.

Let $V_{1}$ and $V_{2}$ be two members of $\mathscr{V}$. Then, $V_{1} \cup V_{2}$ and $V_{1} \cap V_{2}$ are both quasi-compact. Let $t_{i} \in \Gamma\left(V_{i}, \mathscr{L}\right)$ represent $\left.s\right|_{V_{i}}$. The difference of $t_{1}$ and $t_{2}$ restricted to $V_{1} \cap V_{2}$ lifts to a section $u$ of $\mathscr{L}^{\prime}$ as $\mathscr{L}^{\prime}$ is quasi-flabby. By adding $\left.u\right|_{V_{2}}$, to $t_{2}$ we may assume that $t_{1}$ and $t_{2}$ agree on the overlap $V_{1} \cap V_{2}$. We may patch $t_{1}$ and $t_{2}$ together to get a section $t$ of $\Gamma\left(V_{1} \cup V_{2}\right.$, $\mathscr{L})$, which represents $\left.s\right|_{V_{1} \cup V_{2}}$. Hence $V_{1} \cup V_{2} \in \mathscr{V}$ and we are done.

By the same reasoning as Godement's Theorem 4.4.3. $a$, we have the

COROllary 5. Let $\mathscr{L}$ be any quasi-flabby sheaf on a quasi-noetherian space $X$. Then, $H^{i}(X, \mathscr{L})$ is zero for positive $i$.

We will next see that quasi-noetherian spaces have pleasant properties with respect to direct limits of sheaves.

Proposition 6. Let $\left(\mathscr{F}_{\alpha}\right)$ be a direct system of sheaves on a quasi-noetherian space $X$. Then, for any quasi-compact open subset $U$ of $X$, we have a natural isomorphism

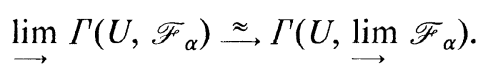

For the proof, one may consult Godement's discussion following his Theorem 3.10.1. Another approach would be to show that the presheaf $\varliminf_{\longrightarrow} \Gamma\left(U, \mathscr{F}_{\alpha}\right)$ satisfies a patching condition for any two quasi-compact open subsets. Thus, one would be able to conclude that this presheaf has the same values as the sheaf $\lim _{\mathscr{F}_{\alpha}}$ for quasi-compact open subsets. The patching condition itself follows from the patching conditions for the $\mathscr{F}_{\alpha}$ and the left exactness of direct limits.

A consequence of the last proposition is the obvious

COROLlary 7. The direct limit of a system of quasi-flabby sheaves on a quasi-noetherian space is always quasi-flabby.

Now, we are ready for the main cohomological consequence of the above discussion. It is

THEOREM 8. Let $\left(\mathscr{F}_{\alpha}\right)$ be a direct system of abelian sheaves on a quasinoetherian space $X$. Then, we have a natural isomorphism

$$
\underset{\longrightarrow}{\lim } H^{i}\left(X, \mathscr{F}_{\alpha}\right) \underset{\approx}{\longrightarrow} H^{i}\left(X, \lim _{\longrightarrow} \mathscr{F}_{\alpha}\right)
$$

for all $i$.

Proof. Let $\mathscr{C}\left(\mathscr{F}_{\alpha}\right)$ denote Godement's canonical flabby sheaf containing $\mathscr{F}_{\alpha}$. We have a direct system of short exact sequences 


$$
0 \rightarrow \mathscr{F}_{\alpha} \rightarrow \mathscr{C}\left(\mathscr{F}_{\alpha}\right) \rightarrow \mathscr{G}_{\alpha} \rightarrow 0 .
$$

Also, we have a limit exact sequence

$$
0 \rightarrow{\underset{\|}{\mathscr{F}}}_{\lim _{\|}} \mathscr{F}_{\alpha} \rightarrow \stackrel{\lim }{\longrightarrow}_{\|} \mathscr{C}\left(\mathscr{F}_{\alpha}\right) \rightarrow \underset{\|}{\rightarrow} \lim _{\mathscr{C}} \mathscr{G}_{\alpha} \rightarrow 0 .
$$

By Corollary $7, \underline{\lim } \mathscr{C}\left(\mathscr{F}_{\alpha}\right)$ is quasi-flabby and, hence, has no higher cohomology by Corollary 5. Using the long exact sequences of cohomology, we have the following commutative exact diagrams:

$$
\begin{aligned}
& 0 \rightarrow \lim _{\longrightarrow} \Gamma\left(X, \mathscr{F}_{\alpha}\right) \rightarrow \lim _{\longrightarrow} \Gamma\left(X, \mathscr{C}\left(\mathscr{F}_{\alpha}\right)\right) \rightarrow \lim _{\longrightarrow} \Gamma\left(X, \mathscr{G}_{\alpha}\right) \rightarrow \lim _{\longrightarrow} H^{1}\left(X, \mathscr{F}_{\alpha}\right) \rightarrow 0 \\
& 0 \rightarrow \quad \Gamma(X, \mathscr{F}) \rightarrow \quad \Gamma(X, \mathscr{C}) \rightarrow \quad \Gamma(X, \mathscr{G}) \rightarrow \quad H^{1}(X, \mathscr{F}) \rightarrow 0
\end{aligned}
$$

and

$$
\begin{aligned}
& 0 \rightarrow \lim _{\longrightarrow} H^{i}\left(X, \mathscr{G}_{\alpha}\right) \rightarrow \lim _{\longrightarrow} H^{i+1}\left(X, \mathscr{F}_{\alpha}\right) \rightarrow 0 \text { for } i>0 \\
& 0 \rightarrow \quad H^{i}\left[(X, \mathscr{G}) \quad \rightarrow \quad H^{i+1}(X, \mathscr{F}) \quad \rightarrow 0 .\right.
\end{aligned}
$$

By induction on $n$, we are done because Proposition 6 included the case $n=0$.

We finish this section by noting

Corollary 9. Let $A$ be a commutative ring and $M$ a flat $A$-module. If $\mathscr{F}$ is a sheaf of A-modules on a quasi-noetherian space $X$, then, there is a natural isomorphism

$$
H^{i}(X, \mathscr{F}) \otimes_{A} M \stackrel{\approx}{\rightarrow} H^{i}\left(X, \mathscr{F} \otimes_{A} M\right) .
$$

Proof. It is true if $M$ is free because cohomology commutes with direct sums. The theorem yields the corollary because every flat module is a direct limit of free ones by Lazard's Theorem [4].

3. Consequences. We will give some application of the theory of last section. Recall that a scheme with a quasi-noetherian underlying topology is a quasi-compact quasi-separated scheme. Furthermore, a morphism $f: X \rightarrow Y$ is quasi-compact and quasi-separated if, for any open affine subvariety $V$ of $Y$, the inverse image $f^{-1} V$ is a quasi-compact quasiseparated scheme.

A direct system of sheaves, which frequently arises, is successive multiplication by a section of the structure sheaf or, more generally, by a section of an invertible sheaf. Let $f$ be a section of an invertible sheaf $\mathscr{L}$ on a scheme $X$. For any $\mathcal{O}_{X}$-module $\mathscr{F}$, we have the direct system $\mathscr{F} \rightarrow \mathscr{F} \otimes$ 
$\mathscr{L} \rightarrow \mathscr{F} \otimes \mathscr{L}^{\otimes 2} \rightarrow \cdots$ given by multiplication by $f$. Denote the limit sheaf by $\lim _{\longrightarrow} \mathscr{F} \otimes \mathscr{L}^{\otimes n}$.

Let $X_{f}$ be the open subscheme of $X$ consisting of points $x$ where $f$ generates the stalk $\mathscr{L}_{x}$ as an $\mathcal{O}_{X}$, -module. Consider the natural $\mathcal{O}_{X^{-}}$ homomorphism $\phi: \mathscr{F} \rightarrow_{X_{f}} \mathscr{F}$. Then $\psi$ induces another $\mathcal{O}_{X}$-homomorphism $\phi: \lim _{\mathscr{F}} \otimes \mathscr{L}^{\otimes n} \rightarrow \lim _{(}\left(X_{f} \mathscr{F}\right) \otimes \mathscr{L}^{\otimes n}$, but the homomorphisms $x_{f} \mathscr{F} \rightarrow\left(x_{f} \widetilde{\mathscr{F}}\right) \otimes \mathscr{L} \rightarrow\left(x_{f} \widetilde{\mathscr{P}}\right) \otimes \mathscr{L}^{\otimes 2} \rightarrow \cdots$ are all isomorphisms. Thus, the composition $\left.x_{f} \mathscr{F} \rightarrow \lim _{\left(X_{f} \mathscr{F}\right.}\right) \otimes \mathscr{L}^{\otimes n}$ is an isomorphism. By this isomorphism, we may regard $\phi$ as an $\mathcal{O}_{X}$-homomorphism $\underline{\lim } \mathscr{F} \otimes \mathscr{L}^{\otimes n} \rightarrow$ $x_{f} \mathscr{F}$.

If $\mathscr{F}$ is a quasi-coherent $\mathcal{O}_{X}$-module, then the natural homomorphism $\lim _{\mathscr{F}} \otimes \mathscr{L}^{\otimes n} \rightarrow{ }_{X_{f}} \mathscr{F}$ is always an isomorphism. Conversely, the quasicoherent $\mathcal{O}_{X}$-modules are exactly the $\mathcal{O}_{X}$-modules which have this property when restricted to any open subscheme of $X$.

With these facts in mind, we may proceed to the proof of

THEOREM 10. [3, 1.4.5] Let $\mathscr{F}$ be a quasi-coherent sheaf on a quasicompact, quasi-separated scheme $X$. Let $\mathscr{L}$ be an invertible $\mathcal{O}_{X}$-module with section $f$. Then, $H^{i}(X, \mathscr{F}) \cong \lim _{\rightarrow} H^{i}\left(X, \mathscr{F} \otimes \mathscr{L}^{\otimes n}\right)$, where the limit is taken over successive multiplication by $f$.

Proof. Let $U$ be $X_{f}$. By Corollary $3, H^{i}(U, \mathscr{F})=H^{i}\left(X, U^{\mathscr{F}}\right)$ because the inclusion $U \subset X$ is affine. On the other hand, ${ }_{U}^{\mathscr{F}}=\lim _{\mathfrak{P}} \otimes \mathscr{L}^{\otimes n}$. The Theorem 8 shows that $H^{i}(X, U \mathscr{F}) \cong \lim _{\longrightarrow} H^{i}\left(X, \mathscr{F} \otimes \mathscr{L}^{\otimes n}\right)$. The theorem follows from these two isomorphisms.

If the invertible sheaf $\mathscr{L}$ is the trivial $\mathcal{O}_{X}$-module $\mathcal{O}_{X}$, then we have the isomorphism $H^{i}\left(X_{f}, \mathscr{F}\right) \approx \lim _{\rightarrow} H^{i}(X, \mathscr{F})$, where the limit is just the localization $H^{i}(X, \mathscr{F})_{(f)}$ of the $\Gamma\left(X, \mathcal{O}_{X}\right)$-module with respect to its element $f$. The translation between localization and limits of successive multiplication is given in E.G.A. 0-1.6.1.

Corollary 11. [3, 1.4.10 or 1.4.11] Let $f: X \rightarrow Y$ be a quasi-compact, quasi-separated morphism and $\mathscr{F}$ a quasi-coherent sheaf on $X$. Then, the higher direct images $R^{i} f_{*} \mathscr{F}$ are quasi-coherent. Further, $\Gamma\left(U, R^{i} f_{*} \mathscr{F}\right) \cong$ $H^{i}\left(f^{-1}(U),\left.\mathscr{F}\right|_{f^{-1}(U)}\right)$ for any affine open subscheme $U$ in $Y$.

Proof. Let $g$ be an element of a commutative $\operatorname{ring} A$. Assume that $Y=$ Spec $A$ and $U=\operatorname{Spec} A_{(g)}$. As $f^{-1} U$ is quasi-compact and quasi-separated, the Theorem 10 gives an isomorphism $H^{i}\left(f^{-1} U, \mathscr{F}\right) \cong \lim _{\longrightarrow} H^{i}(X, \mathscr{F})=$ $\left.H^{i} X, \mathscr{F}\right)_{(g)}$. Thus, $R^{i} f_{*} \mathscr{F}$ must be the quasi-coherent sheaf $H^{i}(X, \mathscr{F})$ associated to the $A$-module $H^{i}(X, \mathscr{F})$. Hence, $\Gamma\left(Y, R^{i} f_{*} \mathscr{F}\right) \cong H^{i}(X, \mathscr{F})$.

We have proven the first statement as it is local on $Y$. The second state- 
ment follows by letting the $U$ of the corollary be the $Y$ of the last paragraph.

THEOREM 12. [3, 1.4.15] Let

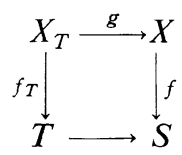

be a cartesian square of schemes, where $f$ is a quasi-compact, quasiseparated morphism. Let $\mathscr{F}$ be a quasi-coherent sheaf on X and $\mathscr{M}$ be quasicoherent on $T$. Assume $\mathscr{M}$ is flat over $S$. Then, the base change mapping.

$$
\left(R^{i} f_{*} \mathscr{F}\right) \bigotimes_{C_{s}} \mathscr{M} \approx R^{i} f_{T_{*}}\left(\mathscr{F} \bigotimes_{\mathcal{O}_{s}} \mathscr{M}\right)
$$

is an isomorphism.

Proof. The question is local on $S$ and $T$. We may assume $S=\operatorname{Spec} A$ and $T=\operatorname{Spec} B$ and $\mathscr{M}=\tilde{M}$, where $B$ is an $A$-algebra and $M$ is a $B$ module, flat over $A$. By corollary 11 , we need to prove that the base change, $H^{i}(X, \mathscr{F}) \otimes_{A} M \stackrel{\approx}{\rightarrow} H^{i}\left(X_{T}, \mathscr{F} \otimes_{A} M\right)$, is an isomorphism. Because $g$ is affine,

$$
H^{i}\left(X_{T}, \mathscr{F} \otimes_{A} M\right) \cong H^{i}\left(X, g_{*}\left(\mathscr{F} \bigotimes_{A} M\right)\right) \cong H^{i}\left(X, \mathscr{F} \otimes_{A} M\right) .
$$

By corollary 9 , the last group is just $H^{i}(X, \mathscr{F}) \otimes_{A} M$.

4. The Čech Cohomology and a Künneth Formula. The proof that I will give for a simple version of the Künneth formula uses Čech resolutions. Let $\mathscr{U}=\left\{U_{i}\right\}$ be a finite open covering of a space $X$. There is a complex $\check{\mathscr{C}}_{\mathscr{U}}^{*}(\mathscr{F})$ with $\check{\mathscr{C}}_{\mathscr{U}}^{n}(\mathscr{F})=\oplus U_{U i_{1} \cap \ldots \cap U_{i_{n}}} \mathscr{F}$, which forms a resolution of $\mathscr{F}, \mathscr{F} \rightarrow \oplus_{U_{i}} \mathscr{F} \rightarrow \cdots$. The complex $\tilde{C}_{\mathscr{U}}^{*}(\mathscr{F})$ of global sections oi $\check{\mathscr{C}}_{\mathscr{U}}^{*}(\mathscr{F})$ has cohomology groups $\check{H}_{\mathscr{U}}^{*}(\mathscr{F})$, the Cech groups.

THEOREM 13. [3, 1.4.1] Let $\mathscr{U}$ be a finite covering of a scheme $X$ by open affines which have affine intersections. If $\mathscr{F}$ is a quasi-coherent sheaf on $X$, then $\check{\mathscr{C}}_{\mathfrak{u}}^{*}(\mathscr{F})$ gives a $\Gamma(X, \quad)$-acyclic resolution of $\mathscr{F}$ by quasi-coherent

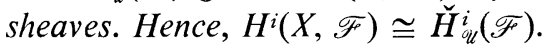

Proof. By assumption, an intersection $V=U_{1} \cap \cdots \cap U_{n}$ is affine and its inclusion in $X$ is an affine morphism. By corollary $3, H^{i}\left(X,{ }_{V} \mathscr{F}\right) \cong$ $H^{i}(V, \mathscr{F})$. By Theorem $1, H^{i}(V, \mathscr{F})$ vanishes if $\mathrm{i}>0$. Hence, ${ }_{V} \mathscr{F}$ is $\Gamma(X, \quad)$-acyclic. Therefore, the direct sum $\check{\mathscr{C}}_{\mathscr{U}}^{n}(\mathscr{F})$ is $\Gamma(X, \quad)$-acyclic. The second fact follows from the first by a standard argument.

This theorem combined with the next gives a relation for computing the cohomology of product sheaves on products in terms of the cohomology 
of the factors. The relationship is called a Künneth formula.

THEOREM 14. [3, 6 ]. Let $X$ and $Y$ be schemes over an affine scheme $S=\operatorname{Spec} A$. Let $\mathscr{F}$ and $\mathscr{G}$ be quasi-coherent sheaves on $X$ and $Y$. Assume that these sheaves are flat over $S$. Let $\mathscr{U}$ and $\mathscr{V}$ be finite open affine covers of $X$ and $Y$ with affine intersections. Then, there are isomorphisms

$$
H^{i}\left(X \times_{S} Y, \mathscr{F} \otimes \mathscr{G}\right)_{S} \approx H^{i}\left(\check{C}_{\mathscr{\mathscr { U }}}^{*}(\mathscr{F}) \bigotimes_{A} \check{C}_{\mathscr{V}}^{*}(\mathscr{G})\right) \text {. }
$$

Furthermore, the $\check{C}$ ech cochains $\check{C}_{\mathscr{U}}^{*}(\mathscr{F})$ and $\check{C}_{\mathscr{V}}^{*}(\mathscr{G})$ are flat A-modules.

Proof. Recall, that, if $Z \rightarrow S$ is an affine scheme over $S$ and $\mathscr{H}$ is a $S$-flat quasi-coherent sheaf on $Z$, then the global sections of $\mathscr{H}$ is a flat $A$-module. The $\breve{C}$ ech cochains $\check{C}_{\mathscr{O}}^{*}(\mathscr{F})$ and $\check{C}_{\mathscr{V}}^{*}(\mathscr{G})$ are $A$-flat as they are direct sums of the above type of $A$-modules. The same reasoning applied locally on $X$ and $Y$ shows that the complexes of sheaves, $\check{\mathscr{C}}_{\mathscr{l}}^{*}(\mathscr{F})$ and $\check{\mathscr{C}}_{\mathscr{V}}^{*}(\mathscr{G})$, consist of $S$-flat quasi-coherent sheaves.

As $\mathscr{F}$ and $\mathscr{G}$ are flat over $S$, we may conclude that $\mathscr{F} \otimes_{S} \mathscr{G}$ has a resolution by the tensor product complex, $\check{\mathscr{C}}_{\mathscr{U}}^{*}(\mathscr{F}) \otimes_{S} \check{\mathscr{C}}_{\mathscr{V}}^{*}(\mathscr{G})$. The remaining points are that this complex gives a $\Gamma\left(X \times_{S} Y\right.$, )-acyclic resolution and the complex of its global sections is just $\check{C}_{\mathscr{Q} /}^{*}(\mathscr{F}) \otimes_{A} \check{C}_{\mathscr{V}}^{*}(\mathscr{G})$.

To see these facts, one looks at each direct summand of $\check{\mathscr{C}}_{\mathscr{V}}^{*}(\mathscr{F}) \otimes_{S}$ $\check{\mathscr{C}}_{V}^{*}(\mathscr{G})$. Each summand has the form ${ }_{U} \mathscr{F} \otimes_{S V} \mathscr{G}$, where $U$ and $V$ are open affines in $X$ and $Y$ and their inclusions in $X$ and $Y$ are affine morphisms. Clearly, ${ }_{U} \mathscr{F} \otimes_{S}{ }_{V} \mathscr{G}$ is isomorphic to ${ }_{U{ }_{S} V} \mathscr{F} \otimes_{S} \mathscr{G}$. As $U \times{ }_{S} V$ is affine and its inclusion in $X \times{ }_{S} Y$ is an affine morphism, we may conclude that ${ }_{U \times{ }_{S} V} \mathscr{F} \otimes_{S} \mathscr{G}$ is a $\Gamma\left(X \times_{S} Y\right)$-acyclic sheaf by the reasoning in the last theorem. Furthermore, the global sections of this sheaf are $\Gamma(U, \mathscr{F}) \otimes_{A}$ $\Gamma(V, \mathscr{G})$, which is the corresponding direct summand of $\check{C}_{\mathscr{U}}^{*}(\mathscr{F}) \otimes_{S} \check{C}_{\mathscr{V}}^{*}(\mathscr{G})$. Hence, $H^{i}\left(X \times{ }_{S} Y, \mathscr{F} \otimes_{S} \mathscr{G}\right)$ is isomorphic to the $i^{\text {th }}$ homology group of the complex $\check{C}_{\mathscr{U}}^{*}(\mathscr{F}) \otimes_{S} \check{C}_{\mathscr{V}}^{*}(\mathscr{G})$.

\section{REFERENCES}

1. Godement, R., Théorie des faisceaux, Actualités scientifiques et industrielles, 1252, Hermann, Paris, 1964.

2. Grothendieck, A., Sur Quelques Points D'Algèbre Homologique, Tōhoku Mathematical Journal, 9 (1957), pp. 119-221.

3. Grothendieck, A., Éléments de Géométrie Algébrique, III, Publications Mathématiques, 11 and 17, Institut des Hautes Etudes Scientifiques, 1961 and 1963.

4. Lazard, D., Autor de la platitude, Bull. Soc. Math. France, 97 (1969), 81-128.

5. Serre, J.-P., Faisceaux algébriques cohérents, Ann. of Math., 61 (-1955), 197-278.

Partly supported by the Sloan Foundation and the N.S.F.

Department of Mathematics The Johns Hopkins University Baltimore, MaryLAND 21218 
